\title{
Using Fluorescence Dyes as a Tool for Analyzing the MALDI Process
}

\author{
Thorsten W. Jaskolla and Michael Karas \\ Institute for Pharmaceutical Chemistry, Johann Wolfgang Goethe University, Frankfurt, Germany
}

In a recent paper (Setz, P. D.; Knochenmuss, R. Phys. Chem. A 2005, 109, 4030-4037) energy-transfer from excited matrix molecules to fluorescent traps was used to study the role of pooling reactions for the ionization processes in matrix-assisted laser desorption ionization (MALDI) using 2,5-dihydroxybenzoic acid as matrix. Exciton trapping was shown to interfere with matrix ionization. These investigations were extended to analyze the influence of fluorescent traps on both matrix and analyte ions for $\alpha$-cyano-4-hydroxycinnamic acid and further matrices. A strong influence of the fluorescent traps on both matrix and analyte ionization was revealed, depending on the matrix:trap ratio, and manifested itself differently for low and high mass analytes. The observations are rationalized by the intermediate formation of a "hot spot" due to an efficient conversion of electronic excitation energy to vibronic energy within the fluorescent traps. This process favors the desorption and ionization of small vaporizable analytes and compromises the cluster ablation process needed for larger analyte ions. (J Am Soc Mass Spectrom 2008, 19, 1054-1061) (C) 2008 American Society for Mass Spectrometry

$\mathrm{M}$ atrix-assisted laser desorption ionization [1-3] (MALDI) has become an enabling technology for the fields of protein mass spectrometry (MS) and proteomics. Despite its widespread use, e.g., for protein identification via peptide mass fingerprinting [4], a comprehensive model for the generation of free gasphase ions has not yet been developed [5, 6]. All matrices in use today have been found empirically and stem from the early days of MALDI, such as $\alpha$-cyano-4-hydroxycinnamic acid (CHCA) [7]. With regard to analyte ionization, the essential role of protonated matrix species was already discussed in the early MALDI papers proposing that the central reaction of analyte ionization in the positive ion mode is a proton transfer from protonated matrix species to analyte molecules. A so-called "photochemical" ionization model was proposed by Ehring et al. [8]; energy pooling processes and resonant two-step absorption were assumed to occur and to facilitate the generation of the primary radical cation matrix species for which more than one UV photon is needed. Karbach et al. experimentally determined the ionization potential of 2,5-DHB to be higher than the energy content of two $337 \mathrm{~nm}$ UV photons [9]. Resonant two-step absorption is only possible taking into consideration that the missing energy is contributed by the thermal energy of the matrix system. This photo/ thermal ionization model was analyzed more in detail by Allwood et al. [10]. Different authors proposed the possibility of energy pooling processes of two neighboring

Address reprint requests to Professor M. Karas, Institute for Pharmaceutical Chemistry, Johann Wolfgang Goethe University, Max von-Laue Str. 9, 60438 Frankfurt, Germany. E-mail: michael.karas@pharmchem.uni-frankfurt.de (localized) excited molecules (multicenter ionization model) $[8,11,12]$ with higher ionization efficiency than resonant two-step absorption processes. This model was further extended by Setz et al. proposing mobile matrix excitations [13].

Laser excitation also induces the MALDI ablation and evaporation process above a distinct threshold fluence. Theoretical investigations of the MALDI process on the basis of the breathing sphere model predict two desorption mechanisms: thermal vaporization of single molecules up to small clusters (four molecules or less) and ablation of large clusters at higher laser fluences [14-16]. The mechanistic discussions and considerations have been carefully reviewed by Dreisewerd [17]. Moreover, it is included in the considerations that the diverging initial velocities as measured for analytes of different mass [18] indicate that small-mass compounds can be desorbed and ionized via a thermal evaporation process with lower $\mathrm{v}_{0}$ values while intact ions of larger and/or thermally labile compounds can only be formed via a cluster emission process characterized by a matrix-dependent higher and uniform initial ion velocity. These assumed differences in the desorption process are analyzed in more detail in this work with the help of fluorescent traps (FTrap) with a lowest excited-state below that of the matrix molecules. FTraps were used to investigate energy pooling and matrix ionization processes; electronic energy transferred to FTraps will be trapped within the additives and is not available for further matrix processes. The quenching of matrix ion yield accompanied by very strong FTrap fluorescence even at very low FTrap 
concentrations (FTrap:matrix $=3 \times 10^{-8}$ ) was presented and discussed as a clear proof that long-range energytransfer within the matrix occurs and contributes to matrix ionization [13]. However, the investigation was restricted to one matrix, 2,5-dihydroxybenzoic acid (DHB), and the presumed effect on analyte ionization was not inspected at all. Here we report on the effect of FTraps on both matrix and analyte ionization and desorption for CHCA and various analytes. Strong effects were observed already at extremely low FTrap concentrations, even below the ratio of $3 \times 10^{-8}$ mentioned above. The results will be discussed including further measurable matrix analyte ion properties, such as mass, initial ion velocity $\left(\mathrm{v}_{0}\right)$ and effective desorption temperature.

\section{Experimental}

\section{Materials and Reagents}

All chemicals were purchased from Sigma-Aldrich Chemical Co. (Munich, Germany) and were analytical grade except when noted otherwise. The matrices ferulic acid (FA), sinapinic acid (SA), 6-aza-2-thiothymine (ATT) were of highest grade possible. The laser dyes 4-(dicyanomethylene)-2-methyl-6-(p-dimethylaminostyryl)$4 \mathrm{H}$-pyran (DCM), pyridine 1 were obtained from Radiant Dyes (Göttingen, Germany). The matrix CHCA was purchased from Bruker Daltonics (Leipzig, Germany). Methanol (MeOH, HPLC-grade) was obtained from Acros (Nidderau, Germany). Acetonitrile (ACN, Rotisolv HPLC-Grade) was purchased from Roth (Karlsruhe, Germany). MilliQ water was prepared by a Millipore (Schwalbach, Germany) water purification system. The Sequazyme Mass Standards Kit (Calibration Mixture 1+2) was obtained from Applied Biosystems (Darmstadt, Germany). P-Methoxybenzylpyridinium chloride was synthesized as described previously [19].

\section{Sample Preparation and Measurement}

The influence of the FTrap concentration was analyzed by mixing $1 \mu \mathrm{L}$ matrix solution (30 mM CHCA, 70\% $\mathrm{ACN}$ ) and $1 \mu \mathrm{l}$ FTrap solution (DCM or pyridine 1 dissolved in ACN) on a polished stainless steel substrate. FTrap dilution series were prepared by stepwise 1:10 dilutions, starting from $3 \mathrm{mM}$ down to $30 \mathrm{fM}$. Additionally, pure matrix solution was spotted without FTraps. After air-drying, both matrix and FTrap ion signal intensities were measured and correlated with the relative matrix to FTrap ratios.

The influence of FTraps on analyte intensities was analyzed by mixing $1 \mu \mathrm{L}$ matrix solution $(30 \mathrm{mM}$ CHCA, $70 \% \mathrm{ACN}$ ), $1 \mu \mathrm{L}$ FTrap solution (DCM or pyridine 1 in $\mathrm{ACN}$ ) and $1 \mu \mathrm{L}$ analyte solution $(\mathrm{N}-$ acetylarginine and Thr-Ser-Lys: $100 \mathrm{nM}$; substance P: 500 nM; ACTH (18-39), hirudin, insulin, lysozyme, neurotensin, and oxidized insulin B-chain: $1 \mu \mathrm{M} ; \alpha-$ lactalbumin, trypsin: $2 \mu \mathrm{M}$; lactoglobulin: $5 \mu \mathrm{M}$; Gln-
Gln: $10 \mu \mathrm{M}$, each in 30\% ACN, 0.1\% TFA; dextran standard 1000: $5 \mu \mathrm{g} / \mu \mathrm{L}$ in water; $\mathrm{NaCl}, \mathrm{KCl}, \mathrm{CsCl}: 400$ $\mathrm{nM}$ in water; cholesterol: $5 \mu \mathrm{M}$ in $\mathrm{ACN})$. After drying at $40{ }^{\circ} \mathrm{C}$ and redissolving in $1.5 \mu \mathrm{L} 50 \% \mathrm{ACN}$, the solution was spotted on a polished stainless steel substrate.

The influence of the matrix was tested by mixing $1 \mu \mathrm{l}$ analyte solution (5 mg dextran standard 1000/mL in water) and $1 \mu \mathrm{L}$ matrix solution ( $3 \mathrm{mg} \mathrm{CHCA} / \mathrm{mL}$ in $70 \% \mathrm{ACN}$ ) with and without $1 \mu \mathrm{L}$ FTrap solution (300 pM pyridine 1 in $\mathrm{ACN}$ ) processed as described above. Different FTraps and alternative additives were tested by mixing $1 \mu \mathrm{L} \mathrm{CHCA} \mathrm{solution} \mathrm{(30} \mathrm{mM}$ in $70 \% \mathrm{ACN}$ ) with $1 \mu \mathrm{L}$ analyte solution (dextran standard 1000, 5 $\mathrm{mg} / \mathrm{mL}$ in water), N-acetylarginine (100 $\mathrm{nM}$ in 30\% ACN, $0.1 \%$ TFA) or $\alpha$-lactalbumin (2 $\mu \mathrm{M}$ in $30 \% \mathrm{ACN}$, $0.1 \%$ TFA) and $1 \mu \mathrm{L}$ of the FTrap solution or alternative additive solution (10 $\mathrm{nM}$ in ACN). The FTrap solutions were freshly prepared for all experiments.

\section{Mass Spectrometry}

Determination of the initial ion velocity values was performed with a Voyager-DE PRO Workstation in linear ion mode. All other mass spectrometric (MS) measurements were carried out using an ABI Voyager DE-STR time-of-flight (TOF) mass spectrometer with $337 \mathrm{~nm}$ nitrogen laser at laser fluences optimized to obtain the highest signal-to-noise $(\mathrm{S} / \mathrm{N})$ ratios. The laser fluences for the analysis of matrix ion intensities were only slightly above threshold to prevent saturation of the ion signals. For comparison of MS measurements with and without addition of FTraps the same laser fluence was used in both experiments. All Voyager DE-STR TOF MS experiments were repeated sixfold with 500 laser shots each. Polished stainless steel targets were used as substrate for all experiments. Voyager DE-STR TOF parameters were as follows for all analytes but proteins: polarity, positive; operation mode, reflector; acceleration voltage, $20 \mathrm{kV}$; grid voltage, $68.5 \%$; delay time, $150 \mathrm{~ns}$; time resolution, $0.5 \mathrm{~ns}$. Using proteins as analytes the settings were as follows: operation mode, linear; acceleration voltage, $25 \mathrm{kV}$; grid voltage, 93.5\%; delay time, $600 \mathrm{~ns}$; time resolution, 1 ns. An advanced baseline correction using Data Explorer ver. 4.5 (Applied Biosystems, Darmstadt, Germany) was performed for all spectra.

\section{Measurement of the Initial Ion Velocity}

In a linear two stage acceleration TOF system $\mathrm{v}_{0}$ of the ablated ions can be determined from the slope of the linear regression of total flight time versus delayed extraction (DE) time $\left(\Delta_{\mathrm{t}}\right.$, total $\left./ \Delta_{\mathrm{DE}}\right)$ using expression eq. $1[18,20]$.

$$
v_{0} \approx \frac{\Delta_{t, \text { total }} / \Delta_{D E}}{t_{\text {cont.extraction }}} \bullet x
$$

" $t_{\text {cont. extraction" }}$ " describes the total flight time using con- 
<smiles>COc1ccc(C[n+]2ccccc2)cc1</smiles>

Scheme 1. The dominating fragmentation pathway of the thermometer ion N-p-methoxybenzylpyridinium ion.

tinuous extraction (DE time $=0 \mathrm{~ns})$ which is determined by the $y$-axis intercept. " $x$ " contains instrumental parameters, e.g., grid $(92 \%)$ and accelerating voltage ( 25 $\mathrm{kV}$ ) and amounts to $0.0692768 \mathrm{~m}$. All measurements were done at optimal laser fluences, resulting in the highest $\mathrm{S} / \mathrm{N}$ ratios. The $\mathrm{DE}$ time was varied in a range from $10 \mathrm{~ns}$ (shortest selectable interval) to $1000 \mathrm{~ns}$ in 200 ns steps; $200 \pm 20$ ns must be added to the preselected DE time due to an instrumental dead time. For each DE time the average flight time of the protonated analyte ions was determined using data from six individual measurements accumulated of 50 shots each. Peak labeling was done at $50 \%$ peak centroid after smoothing the spectra using the Savitzky-Golay method with 251 data points. CHCA was used as matrix for all $\mathrm{v}_{0}$ measurements.

\section{Measurement of Effective Plume Temperature}

The laser fluence dependent effective temperature $\left(\mathrm{T}_{\text {eff }}\right)$ as a measure for the internal ion energy was measured using the thermometer molecule N-p-methoxybenzylpyridinium $\left(\mathrm{M}^{+}\right)$chloride. The ion $\mathrm{M}^{+}$exhibits a simple fragmentation pattern with only one intense fragment ion $\left(\mathrm{F}^{+}\right)$(Scheme 1).

Determination of the ion peak areas of $\mathrm{M}^{+}$and $\mathrm{F}^{+}$ allows for the calculation of the survival yield SY $=$ $\mathrm{I}\left(\mathrm{M}^{+}\right) /\left[\mathrm{I}\left(\mathrm{M}^{+}\right)+\mathrm{I}\left(\mathrm{F}^{+}\right)\right]$. This affords a correlation of the SY with $\mathrm{T}_{\text {eff }}$ by calculated dissociation rate constants from well studied fragmentation processes using RiceRamsperger-Kassel-Marcus (RRKM) models [19, 21].
The reliable $\mathrm{T}_{\text {eff }}$ range of $\mathrm{M}^{+}$ranges from 800 to $1500 \mathrm{~K}$ which is in good agreement with the detected effective temperatures. Lower or higher temperature values lead to reduced accuracy caused by too low intensities of $\mathrm{F}^{+}$ or $\mathrm{M}^{+}$. The monoisotopic areas of $\mathrm{M}^{+}$and its fragment $\mathrm{F}^{+}$were determined for $\mathrm{CHCA}, \mathrm{ATT}, \mathrm{SA}$, and also with 400 attomol pyridine 1 added to each of the three matrices. Effective temperatures were calculated based on the correlating SYs. All measurements were repeated six times, each accumulated from 500 shots. For every measurement a new sample spot was used. Laser fluences slightly above the threshold fluence are applied to minimize interfering matrix ion signals.

\section{Results and Discussion}

The strong impact of even very low FTrap-to-matrix ratios on the CHCA matrix ion signals is depicted in Figure 1 and 2. Both FTraps induce a significant and uniform reduction of all major matrix signals (e.g., $\left.\mathrm{MH}^{+}, 2 \mathrm{MH}^{+}, \mathrm{MH}^{+}-\mathrm{H}_{2} \mathrm{O}\right)$ when added at a relative ratio of $1 \times 10^{-12}$ to $1 \times 10^{-10}$ to the matrix. Also the radical matrix molecular cation follows the same trend. Increasing the FTrap amount leads to a partial or full recovery of the matrix signals with maximum matrixion signal intensities at a FTrap-to-matrix ratio of $1 \times 10^{-8}$ for pyridine 1 and $1 \times 10^{-7}$ for DCM. It is striking that a strong effect of the FTraps is observed in a range when FTrap signals $\left(\mathrm{DCMH}^{+}\right.$and $\left.\mathrm{DCM}^{+}\right)$are not yet detected. Respective signals are detected starting at about $100 \mathrm{fmol}$ (FTrap-to-matrix ratio of $3.3 \times 10^{-6}$ ) which is accompanied first by a gradual decrease in matrix-ion signals and finally by a strong drop of matrix-ion signals presumably due to an impairment of the MALDI process when the FTrap amount is larger than about $1 \%$ of the matrix amount.

In the next step the effect of the added FTraps on analyte signals was investigated. Figure 3 summarizes the influence of the dye pyridine 1 on two different

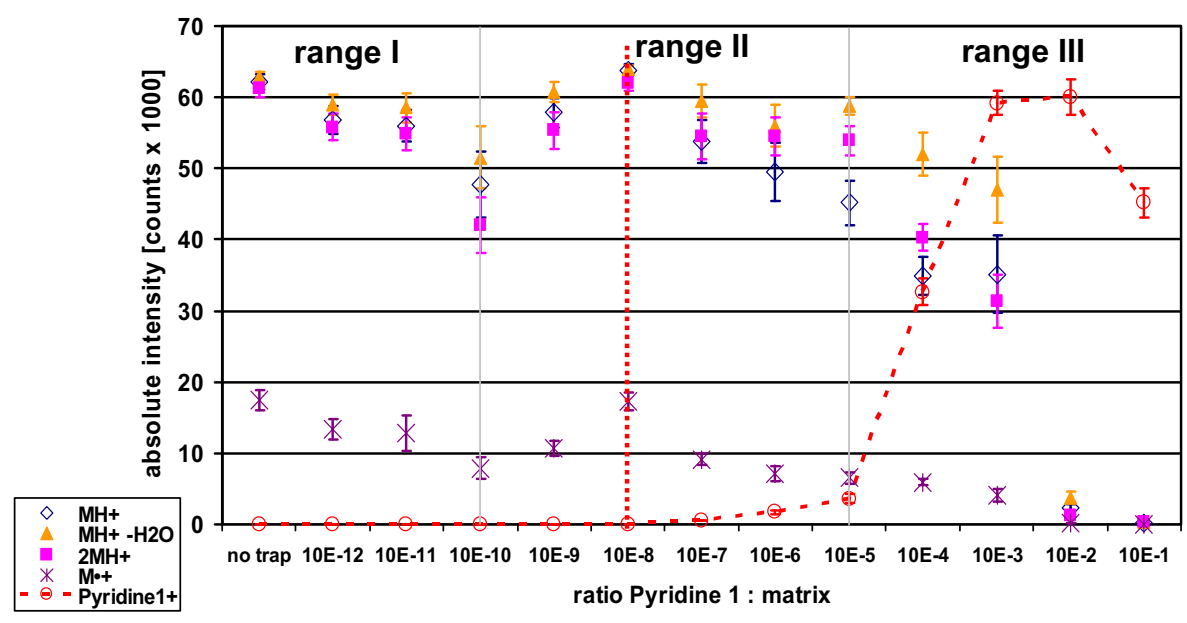

Figure 1. Absolute intensities of several CHCA matrix $(\mathrm{M})$ ions as a function of the pyridine 1-to-matrix ratio. The vertical dashed line indicates the trap amount with the strongest influence. 


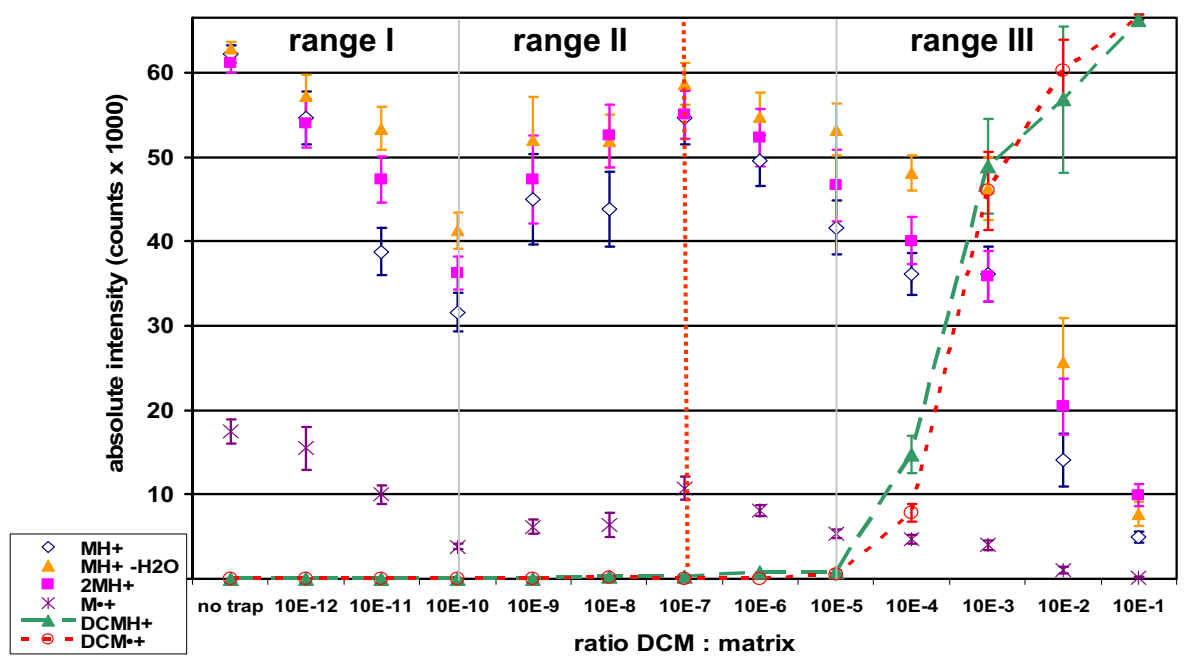

Figure 2. Absolute intensities of protonated DCM, DCM radical cation, and of several CHCA matrix (M) ions as function of the DCM-to-matrix ratio. The vertical dashed line indicates the trap amount with the strongest influence.

analytes, $\mathrm{N}$-acetylarginine and the small protein $\alpha$ lactalbumin. While a strong decrease of the signal intensity is observed for $\alpha$-lactalbumin in the FTrap-to-matrix range between 0 and $1 \times 10^{-8}$ with some recovery of the signal at higher relative FTrap ratios, $\mathrm{N}$-acetylarginine is not strongly affected by the addition of very low FTrap amounts, but shows a maximum of its protonated signal at a FTrap-to-matrix ratio of $1 \times 10^{-8}$, i.e., at an added FTrap amount when the matrix signals reach their maximum. To investigate the indicated mass effect in more detail a series of analytes was inspected ranging from $\mathrm{N}$-acetylarginine and di- and tripeptides to peptides and small proteins up to trypsin. Figure 4 summarizes the results for the FTrap DCM at a FTrap-tomatrix ratio of $3 \times 10^{-6}$.

While all low-mass analytes show an increase in their signal intensity as observed for $\mathrm{N}$-acetylarginine, a transition to signal decrease is observed in the peptide mass range. This occurs at about $1500 \mathrm{Da}$ (a logarithmic plot for the mass range from 0 to $6000 \mathrm{Da}$ is given in Supplementary Figure 1).

In a further experiment we verified that the detected effects caused by FTraps do not occur due to other reasons, e.g., simple structural disturbance of the crystal lattice. For this purpose additives like phloroglucinol, hydroquinone, anthracen-9-carboxylic acid, SA or FA were added to the matrix preparation in low amounts (molar ratio $3.3 \times 10^{-7}$ ). Dextran standard 1000, Nacetylarginine and $\alpha$-lactalbumin were used as analytes. In all preparations analyte ion intensities stayed constant within the experimental error compared to additivefree reference approaches (Supplementary Figure 2-4). Therefore the effects described above cannot be due to matrix doping with passive compounds causing crystal

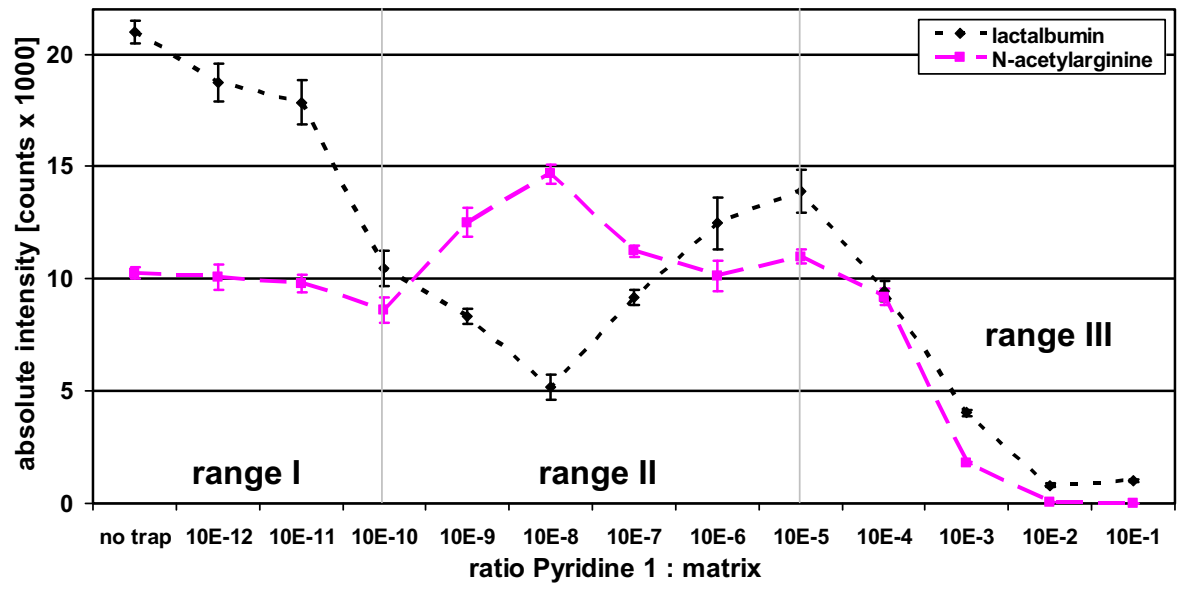

Figure 3. Absolute intensities of high and low mass analytes ( $\alpha$-lactalbumin: $14.2 \mathrm{kDa}, \mathrm{N}$ acetylarginine: $216 \mathrm{Da}$ ) depending on the pyridine 1-to-matrix ratio. Relative matrix-to-analyte ratios were $3 \times 10^{5}$ ( $\mathrm{N}$-acetylarginine) and $1.5 \times 10^{4}$ ( $\alpha$-lactalbumin), respectively. Both analytes were measured from independent samples. 


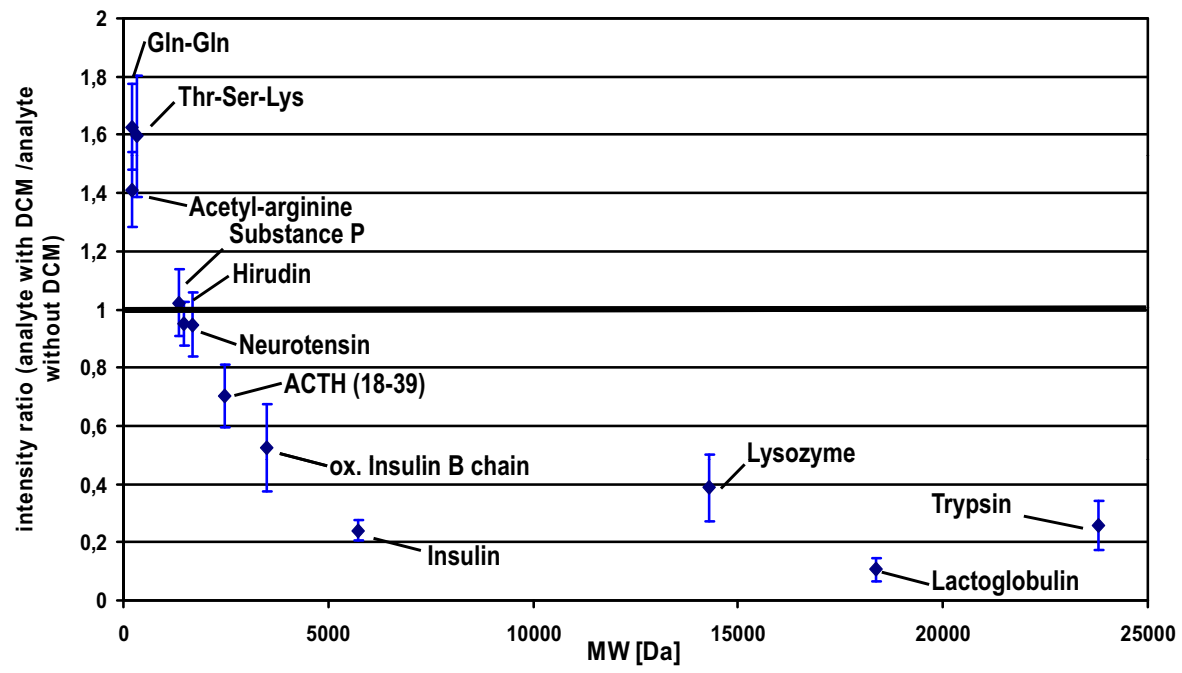

Figure 4. Peptide and protein intensity ratios for samples containing a relative DCM ratio of $3 \times 10^{-6}$ versus nondoped control samples.

defects. The possibility of surface adsorption of FTraps on the matrix crystals was checked by washing the doped matrix crystals. No differences in analyte intensity could be observed in agreement to the results reported in [13].

To elucidate the underlying reasons for the FTrap effects further experiments were carried out. When inspecting the matrix mass spectra a strong increase in alkaline cation and in alkaline cation adduct signal intensities was observed when FTraps were added in amounts to yield the maximum of matrix ion signals. At the same time a strong increase of cationized analyte signals is observed, e.g., for cholesterol or oligosaccharides (dextran 1000 standard). This is depicted in Figures 5 and 6; salt contamination by the added FTrap as reason for the detected increase in analyte ion intensity can be ruled out because of its extreme dilution. The lack of gain in the cholesterol $\mathrm{K}^{+}$-signal may be due to an insufficient amount of potassium present in the sample.

For the case of the dextran standard, addition of FTraps enhances the signal intensities in general, but also induces a shift in the intensity distribution to smaller oligomers (Figure 7); a shift can also be induced by enhanced laser fluence for the FTrap-free sample.

Since the initial velocity of analyte ions was proposed as a means to differentiate between different MALDI desorption processes [22], $\mathrm{v}_{0}$ was determined for a series of peptide analytes from des-arg ${ }^{1}$-bradykinin to bovine insulin. While the larger peptides showed no decrease in their $\mathrm{v}_{0}$ values, data for des-arg-bradykinin and angiotensin I showed a substantial reduction for both dyes (Table 1) in agreement with the transition point found before (Supplementary Figure 1); the effect is the stronger the smaller the analyte mass is.

This observation as well as the increase in small peptide ion and alkaline cation signals raised the question whether an increase in the effective temperature for the MALDI process can be determined via fragmenta- tion of thermometer molecules. Indeed a small but significant temperature rise between 50 and $70 \mathrm{~K}$ was observed for CHCA and also for other tested matrices, like ATT and SA (see Table 2). Since $\mathrm{T}_{\text {eff }}$ determination
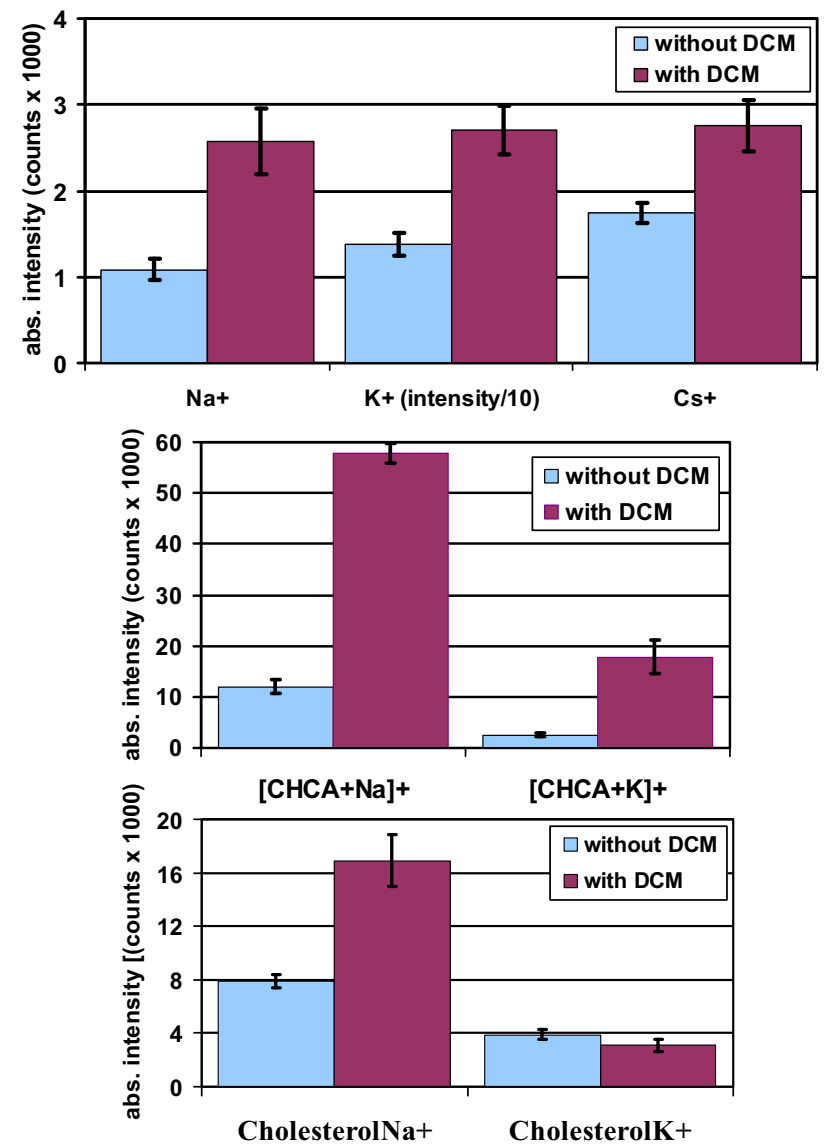

Figure 5. Intensity increase detected for different small analytes for a sample containing DCM at a relative ratio of $3.3 \times 10^{-7}$. Salts (top), matrix salt adducts (middle), and cationized cholesterol (bottom); the potassium ion intensity was divided by 10 . 

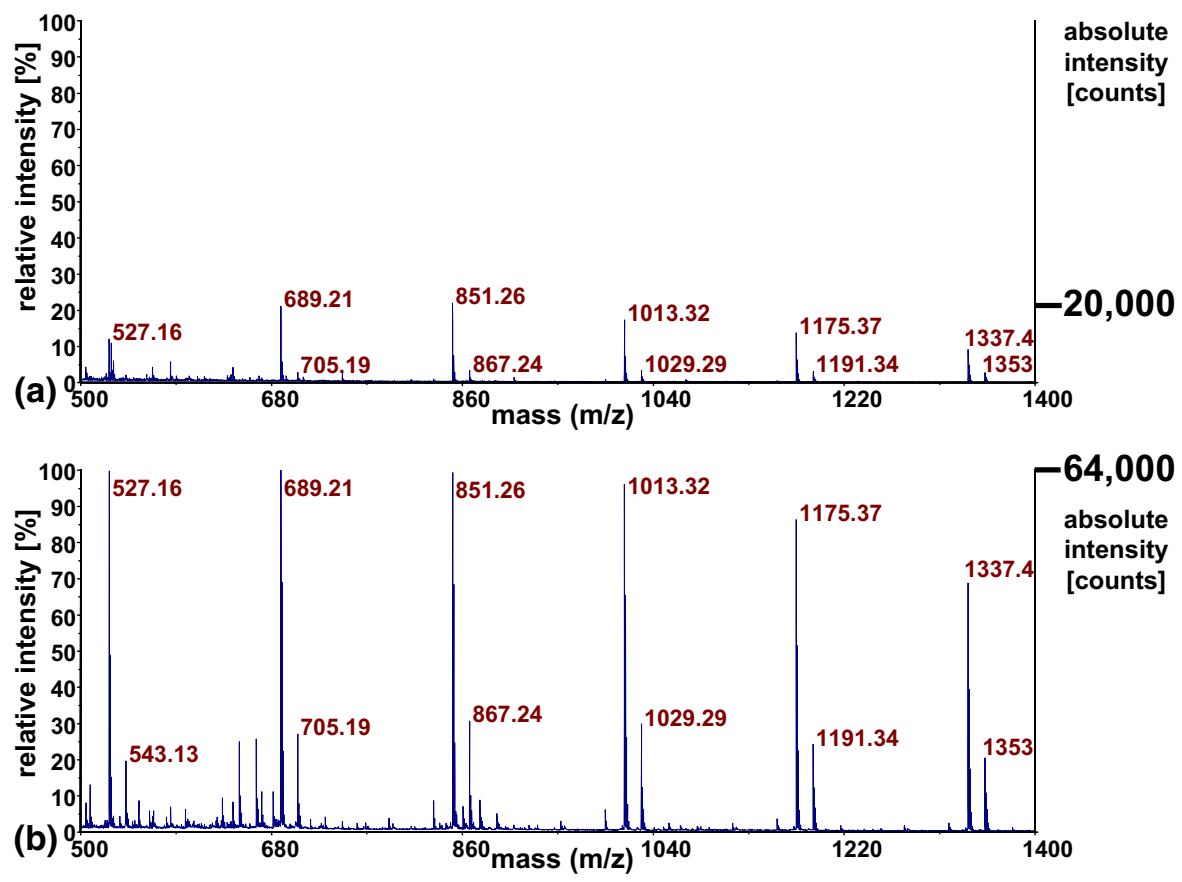

Figure 6. MALDI mass spectra of dextran standard $1000(5 \mu \mathrm{g})$ (a) control, (b) after addition of pyridine 1 at a relative ratio of $1 \times 10^{-7}$. Signals of sodiated and potassiated glucose oligomers are labeled.

is carried out close to threshold fluence for preventing matrix interference, the temperature rise at the higher fluence used for optimal analyte detection is assumed to be even larger.

To interpret and rationalize the above observations a simplified model will be used which includes the following key considerations: laser irradiation results in the excitation of matrix molecules and via energy pooling processes in the formation of matrix radical molecular cations; direct excitation of the dyes plays only a minor role due to their low absorption at the laser wavelength which was indeed a selection criterion used in [13]. Matrix radical cations form the starting point for secondary reactions within matrix clusters or the resulting high-density gaseous plume of material. Matrix ions formed, especially Matrix $\mathrm{H}^{+}$-ions, are the reagent ions within a (photo)chemical ionization process, leading to protonated analyte species by proton transfer reactions $[6,8]$. Analyte ionization reactions either occur via collisions between ion and neutral monomers in the

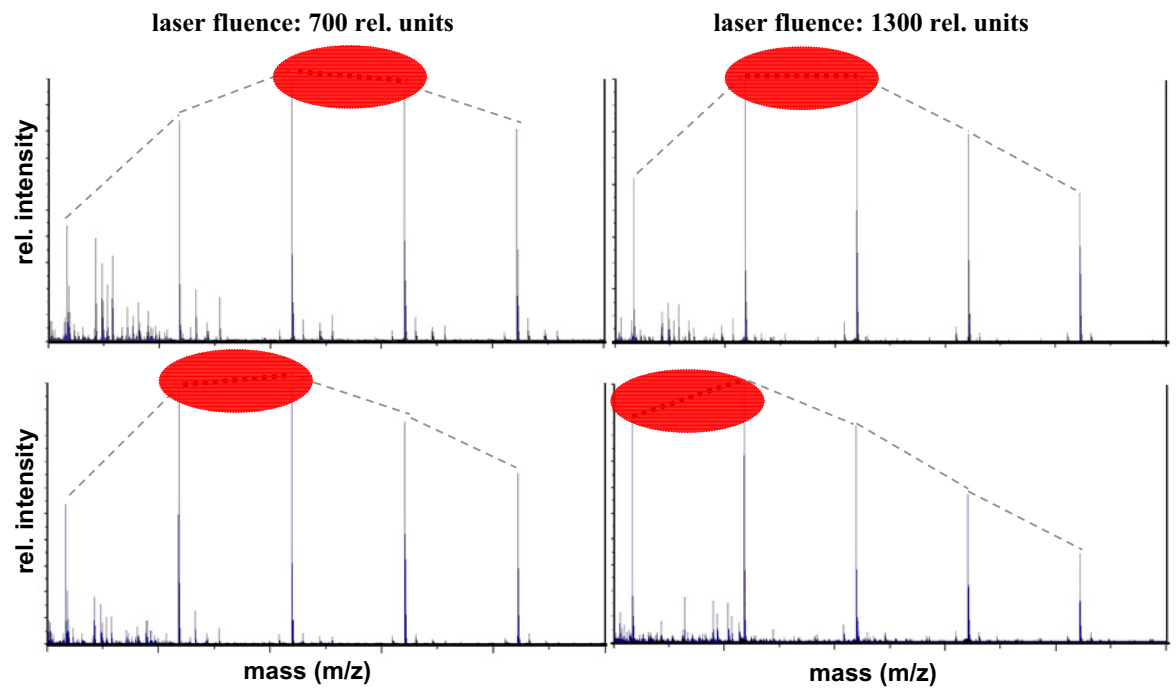

Figure 7. MALDI mass spectra of dextran standard $1000(5 \mu \mathrm{g})$ for different laser fluences with and without pyridine 1 . Top: reference, bottom: addition of pyridine 1 at a relative ratio of $1 \times 10^{-8}$; the two most prominent oligosaccharide ion signals are marked. 
Table 1. Initial velocities $\mathrm{v}_{0}$ of different analytes with and without addition of DCM (relative ratio $1 \bullet 10^{-7}$ ) and pyridine 1 (relative ratio $\left.1 \cdot 10^{-8}\right)$, respectively

\begin{tabular}{|c|c|c|c|c|c|c|c|}
\hline & $\begin{array}{l}\text { des-Arg'- } \\
\text { bradykinin }\end{array}$ & $\begin{array}{l}\text { Angio- } \\
\text { tensin I }\end{array}$ & $\begin{array}{l}\text { Glu'-fibrino- } \\
\text { pep. B }\end{array}$ & $\begin{array}{l}\text { ACTH } \\
(1-17)\end{array}$ & $\begin{array}{c}\text { ACTH } \\
(18-39)\end{array}$ & $\begin{array}{l}\text { ACTH } \\
(7-38)\end{array}$ & $\begin{array}{c}\text { Insulin } \\
\text { (bovine) }\end{array}$ \\
\hline $\begin{array}{l}m / z_{\text {mono }}\left(\mathrm{AH}^{+}\right) \\
\text {preparation }\end{array}$ & 904.5 & 1296.7 & $\begin{array}{l}1570.7 \\
\text { initial velc }\end{array}$ & $\begin{array}{c}2093.1 \\
\mathrm{~m} / \mathrm{s}] \text { of }[\mathrm{ar}\end{array}$ & $\begin{array}{c}2465.2 \\
+\mathrm{H}]^{+}\end{array}$ & 3657.9 & 5730.6 \\
\hline CHCA & $255 \pm 13$ & $304 \pm 11$ & $276 \pm 12$ & $289 \pm 23$ & $281 \pm 11$ & $323 \pm 5$ & $308 \pm 10$ \\
\hline$+\mathrm{DCM}$ & $196 \pm 5$ & $226 \pm 4$ & $286 \pm 20$ & $281 \pm 11$ & $262 \pm 9$ & $287 \pm 19$ & $282 \pm 12$ \\
\hline + Pyridine 1 & $168 \pm 3$ & $201 \pm 4$ & $263 \pm 4$ & $289 \pm 4$ & $292 \pm 5$ & $321 \pm 5$ & $312 \pm 8$ \\
\hline
\end{tabular}

gas phase or within clusters that form the reaction vials for the proton-transfer ionization reactions as discussed in [5].

As described in detail in [13], energy-transfer from excited matrix states to lower-lying excited states of FTraps by exciton hopping is a possible, effective and fast process. Irrespective of the fate of the trapped energy, the loss of excitation energy within the matrix system will quench the ionization of the matrix compared to the undisturbed energy pooling process. Electronic energy trapped in FTraps can be released by fluorescence, internal conversion (IC), photodissociation or intersystem crossing (ISC). Deactivation by means of ISC has only a very low probability, e.g., no greater than 0.01 for DCM [23]. DCM molecules undergo limited photoionization, as indicated by the lack of radical cation signals below amounts of $\sim 100 \mathrm{fmol}$. This agrees with its application as laser dye, which requires high photostability. Pyridine 1 behaves differently, since it contains a quaternary ammonium function and its even-electron ion [pyridine 1] ${ }^{+}$does not reflect photoionization processes. The decrease in [pyridine 1$]^{+}$signal intensities at high dye amounts is therefore contributed to an increasing impairment of the desorption ionization process (see Figure 1). Therefore the dominating energy releasing pathways will be fluorescence and IC. Fluorescence would only induce a loss of energy otherwise available for desorption and ionization, which could be overcome by an increase in laser fluence; however, while all comparisons between FTrap-free and -doped samples were carried out at identical laser fluences, increase in laser fluence for doped samples did not lead to a complete recovery of high mass ion signal intensities.

In the undoped matrix-analyte system IC of electronic energy of excited matrices results in a uniform thermal excitation of the irradiated matrix crystal surface inducing the cluster ablation process needed for the detection of large analytes. For the doped system, we suppose that IC within the FTraps results in localized "hot spots" and reduces the average electronic excitation of the matrix. The prerequisite for the generation of a hot spot is a relatively slow heat conduction-a time constant of about $10 \mathrm{~ns}$ was approximated for the DHB system [17] —while internal conversion occurs within ps and energy-transfer via hopping was estimated to occur within 50 ps [13], facilitating even multiple trapping and conversion steps. The lower the fluorescence quantum yield of the FTrap, the stronger the IC and, hence, the increase in effective temperature will be. The decrease of matrix ion signals and especially of high mass compounds in the very low FTrap concentration range (range I in Figures 1 to 3 ) is attributed firstly to a quenching of the matrix ionization by reducing exciton density. Secondly, a growing impairment of the cluster ablation process occurs by the increasing amount of excitation energy transferred into localized hot spots. Presumably, also losses of energy into deeper regions of the matrix crystals that are usually not affected by one laser shot occur.

Range II exhibits a more complicated behavior. While the decrease of high mass analytes proceeds, both matrix and small mass analyte signals recover and reach a maximum at a relative ratio of $1 \times 10^{-8}$ for pyridine 1 and $1 \times 10^{-7}$ for DCM, respectively. The mass dependence for the analytes is clearly shown in Figure 4. With respect to the data presented in [13] and taking into consideration that different matrices were used one can state that there is at least a qualitative agreement, since the measurements in [13] do not refer to the extreme dilution region (range I); the doping experiments start at FTrap amounts which correlate to the maximum observed in range II. Including the additional results reported above, i.e., a decrease of the initial ion velocity for low mass analytes and an increase in the effective temperature, this is attributed to

Table 2. Survival yields (SY), effective temperatures ( $\mathrm{T}_{\mathrm{eff}}$ ), and temperature increases for CHCA, ATT, and SA with and without addition of pyridine 1 (relative ratio $1.3 \cdot 10^{-8}$ ) at laser fluences slightly above threshold fluence

\begin{tabular}{|c|c|c|c|c|c|c|}
\hline & \multicolumn{2}{|c|}{$\begin{array}{c}\text { CHCA laser intensity: } 350 \\
\text { units }\end{array}$} & \multicolumn{2}{|c|}{$\begin{array}{l}\text { ATT laser intensity: } 1100 \\
\text { units }\end{array}$} & \multicolumn{2}{|c|}{$\begin{array}{c}\text { SA laser intensity: } 1100 \\
\text { units }\end{array}$} \\
\hline & SY [\%] & $\mathrm{T}_{\mathrm{eff}}[\mathrm{K}]$ & SY [\%] & $\mathrm{T}_{\text {eff }}[\mathrm{K}]$ & SY [\%] & $\mathrm{T}_{\mathrm{eff}}[\mathrm{K}]$ \\
\hline Without pyridine1 & $74.0 \pm 2.7$ & $1022 \pm 11$ & $83.8 \pm 2.3$ & $975 \pm 14$ & $67.4 \pm 4.5$ & $1050 \pm 18$ \\
\hline With pyridine 1 & $60.2 \pm 2.3$ & $1080 \pm 10$ & $69.1 \pm 3.0$ & $1044 \pm 13$ & $56.6 \pm 1.6$ & $1095 \pm 6$ \\
\hline$\Delta \mathrm{T}$ & \multicolumn{2}{|c|}{$+58 \mathrm{~K}$} & \multicolumn{2}{|c|}{$+69 \mathrm{~K}$} & \multicolumn{2}{|c|}{$+45 \mathrm{~K}$} \\
\hline
\end{tabular}


an increasingly effective contribution of thermal desorption processes via localized hot spots around the FTraps at the expense of cluster ablation processes. With respect to hot spots the determined increase in $\mathrm{T}_{\text {eff }}$ values cannot be directly correlated to the local temperature rise, since the measurement delivers an average over the whole desorbed volume. The strong effect of enhanced thermal desorption shows up especially for the matrix signals. While photochemical reaction products only reach their initial (undoped) signal intensities, cationized species exhibit a stronger enhancement yield (Figures 1, 2, and 5); this is rationalized by the fact that cationization is not affected by the quenching of radical precursor ions induced by FTraps. Matrices, as well as small mass analytes, can undergo increased thermal vaporization; cationization in the gas phase leads to high intensity alkaline adduct ions. In the case of small mass analytes the increase in thermal desorption outbalances the loss of analyte transfer into gas-phase due to decreasing cluster ablation whereas analyte ions with high masses lose intensity due to decreased cluster ablation processes. The emphasis between thermal desorption and cluster ablation gradually switches with the analyte mass, which can be clearly seen in the linear plot in Supplementary Figure 1.

The inverse trend observed for low (including matrix) and high mass analyte ions in range II (Figure 3 ) is rationalized by the consideration that at increasingly high trap concentrations the energy transferred to each single trap decreases. Therefore, hot-spot formation is less pronounced. Accordingly, a lower thermal vaporization with a decrease in intensity of small thermally vaporizable analytes occurs. The increasing number of FTraps undergoing IC reactions, however, results in a more uniform excitation of the solid, similar to matrix IC reactions in FTrap free approaches, and cluster ablation processes intensify again. Consequently, the intensity of large cluster-ablated analytes like $\alpha$-lactalbumin recovers. Finally, when FTrap amounts reach a relative ratio of $\sim 1 \times 10^{-2}$ to $1 \times 10^{-3}$, a successful MALDI process is impaired resulting in a steep drop of both matrix and analyte signals (end of range III).

\section{Conclusions}

We have shown that the addition of extremely low amounts of FTraps can significantly influence the MALDI process. The lowest excited-state of the FTraps lies below that of the matrix molecules, which causes a trapping of electronic energy from excited matrix states to FTraps by long-range energy-hopping [13]. As a consequence, the matrix crystal system loses electronic energy, which results in a decrease of photoionization products and cluster ablation processes. These FTrap amounts, even though very low, induce a substantial reduction in the signal intensities of high-mass analytes. The energy will be converted mainly into localized vibronic excitation and fluorescence by the FTraps leading to local hot spots surrounding the FTraps. Small thermally vaporizable molecules such as low mass peptides, salts, lipid, or sugars benefit from these processes and are vaporized more easily, resulting in higher ion intensities than in nondoped systems when optimized FTrap amounts are used. In agreement with the measured temperature rise, decreased $\mathrm{v}_{0}$ values are detected for small thermally vaporizable molecules. Therefore, the use of FTraps in doping amounts in MALDI opens a possibility for converting energy within the matrix system and gives an explanation for the different desorption processes and analyte-mass dependent $\mathrm{v}_{0}$ values described in literature.

\section{References}

1. Karas, M.; Bachmann, D.; Hillenkamp, F. The Influence of the Wavelength in High Irradiance Ultraviolet Laser Desorption Mass Spectrometry of Organic Molecules. Anal. Chem. 1985, 57, 2935-2939.

2. Karas, M.; Bachmann, D.; Bahr, U.; Hillenkamp, F. Matrix-Assisted Ultraviolet Laser Desorption of Nonvolatile Compounds. Int. J. Mass Spectrom. Ion Processes 1987, 78, 53-56.

3. Karas, M.; Hillenkamp, F. Laser Desorption Ionization of Proteins with Molecular Masses Exceeding 10,000 Dalton. Anal. Chem. 1988, 60, 22992301.

4. Henzel, W. J.; Watanabe, C.; Stults, J. T. Protein Identification: The Origins of Peptide Mass Fingerprinting. J. Am. Soc. Mass Spectrom. 2003, 14, 931-942.

5. Krüger, R.; Karas, M. Ion Formation in MALDI: The Cluster Ionization Mechanism. Chem. Rev. 2003, 103, 427-440.

6. Knochenmuss, R.; Zenobi, R. MALDI Ionization: The Role of In-Plume Processes. Chem. Rev. 2003, 103, 441-452.

7. Beavis, R. C.; Chaudhary, T.; Chait, B. T. $\alpha$-Cyano-4-hydroxycinnamic Acid as a Matrix for Matrix-Assisted Laser Desorption Mass Spectrometry. Org. Mass Spectrom. 1992, 27, 156-158.

8. Ehring, H.; Karas, M.; Hillenkamp, F. Role of Photoionization and Photochemistry in Ionization Processes of Organic Molecules and Relevance for Matrix-Assisted Laser Desorption Ionization Mass Spectrometry. Org. Mass Spectrom. 1992, 27, 472-480.

9. Karbach, V.; Knochenmuss, R. Do Single Matrix Molecules Generate Primary Ions in Ultraviolet Matrix-Assisted Laser Desorption/ Ionization. Rapid Commun. Mass Spectrom. 1998, 12, 968-974.

10. Allwood, D. A.; Dyer, P. E.; Dreyfus, R. W. Ionization Modeling of Matrix Molecules in Ultraviolet Matrix-Assisted Laser Desorption/ Ionization. Rapid Commun. Mass Spectrom. 1997, 11, 499-503.

11. Liao, P.-C.; Allison, J. Ionization Processes in Matrix-Assisted Laser Desorption/Ionization Mass Spectrometry: Matrix-Dependent Formation of $[\mathrm{M}+\mathrm{H}]^{+}$vs. $[\mathrm{M}+\mathrm{Na}]^{+}$Ions of Small Peptides and Some Mechanistic Comments. J. Mass Spectrom. 1995, 30, 408-423.

12. Knochenmuss, R.; Dubois, F.; Dale, M. J.; Zenobi, R. The Matrix Suppression Effect and Ionization Mechanisms in Matrix-Assisted Laser Desorption/Ionization. Rapid Commun. Mass Spectrom. 1996, 10, 871-877.

13. Setz, P. D.; Knochenmuss, R. Exciton Mobility and Trapping in a MALDI Matrix. J. Phys. Chem. A 2005, 109, 4030-4037.

14. Zhigilei, L. V.; Kodali, P. B. S.; Garrison, B. J. Molecular Dynamics Model for Laser Ablation and Desorption of Organic Solids. J. Phys. Chem. B 1997, 101, 2028-2037.

15. Zhigilei, L. V.; Kodali, P. B. S.; Garrison, B. J. A Microscopic View of Laser Ablation. J. Phys. Chem. B 1998, 102, 2845-2853.

16. Zhigilei, L. V.; Garrison, B. J. Microscopic Mechanisms of Laser Ablation of Organic Solids in the Thermal and Stress Confinement Irradiation Regimes. J. Appl. Phys. 2000, 88, 1281-1298.

17. Dreisewerd, K. The Desorption Process in MALDI. Chem. Rev. 2003, 103, 395-425.

18. Glückmann, M.; Karas, M. The Initial Ion Velocity and Its Dependence on Matrix, Analyte, and Preparation Method in Ultraviolet MatrixAssisted Laser Desorption/Ionization. J. Mass Spectrom. 1999, 34, 467-477.

19. Gabelica, V.; Schulz, E.; Karas, M. Internal Energy Build-Up in MatrixAssisted Laser Desorption/Ionization. J. Mass Spectrom. 2004, 39, 579-593.

20. Juhasz, P.; Vestal, M. L.; Martin, S. A. On the Initial Velocity of Ions Generated by Matrix-Assisted Laser Desorption Ionization and Its Effect on the Calibration of Delayed Extraction Time-of-Flight Mass Spectra. J. Am. Soc. Mass Spectrom. 1997, 8, 209-217.

21. Luo, G.; Marginean, I.; Vertes, A. Internal Energy of Ions Generated by Matrix-Assisted Laser Desorption/Ionization. Anal. Chem. 2002, 74, 61856190.

22. Karas, M.; Bahr, U.; Fournier, I.; Glückmann, M.; Pfenninger, A. The Initial-Ion Velocity as a Marker for Different Desorption-Ionization Mechanisms in MALDI. Int. J. Mass Spectrom. 2003, 226, 239-248.

23. Turban, A. A.; Bondarev, S. L.; Knyukshto, V. N.; Stupak, A. P. Quenching of Fluorescence for Fluoro Derivatives of the Laser Dye DCM in Polar Solutions. J. Appl. Spectros. 2006, 73, 678-685. 